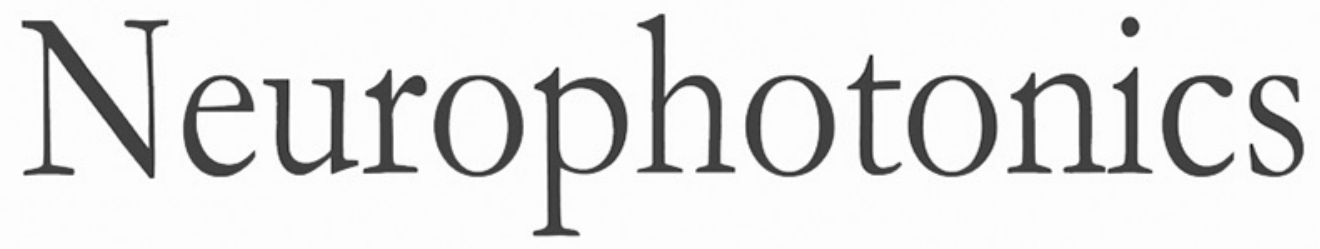

\title{
Acute administration of methylphenidate differentially affects cortical processing of emotional facial expressions in attention-deficit hyperactivity disorder children as studied by functional near-infrared spectroscopy
}

Megumi Kobayashi

Takahiro Ikeda

Tatsuya Tokuda

Yukifumi Monden

Masako Nagashima

Sakae G. Mizushima

Takeshi Inoue

Keiichi Shimamura

Yuta Ujiie

\author{
Akari Arakawa \\ Chie Kuroiwa \\ Mayuko Ishijima \\ Yuki Kishimoto \\ So Kanazawa \\ Takanori Yamagata \\ Masami K. Yamaguchi \\ Ryoichi Sakuta \\ Ippeita Dan
}

Megumi Kobayashi, Takahiro Ikeda, Tatsuya Tokuda, Yukifumi Monden, Masako Nagashima, Sakae G. Mizushima, Takeshi Inoue, Keiichi Shimamura, Yuta Ujiie, Akari Arakawa, Chie Kuroiwa, Mayuko Ishijima, Yuki Kishimoto, So Kanazawa, Takanori Yamagata, Masami K. Yamaguchi, Ryoichi Sakuta, Ippeita Dan, "Acute administration of methylphenidate differentially affects cortical processing of emotional facial expressions in attention-deficit hyperactivity disorder children as studied by functional near-infrared spectroscopy," Neurophoton. 7(2), 025003 (2020), doi: 10.1117/1.NPh.7.2.025003 


\title{
Acute administration of methylphenidate differentially affects cortical processing of emotional facial expressions in attention-deficit hyperactivity disorder children as studied by functional near-infrared spectroscopy
}

\author{
Megumi Kobayashi, ${ }^{a, b, *}$ Takahiro Ikeda,, ${ }^{\text {,cc }}$ Tatsuya Tokuda,,d \\ Yukifumi Monden, ${ }^{b, c, d, e ~}$ Masako Nagashima, ${ }^{\text {b,c }}$ Sakae G. Mizushima,,d, \\ Takeshi Inoue, ${ }^{b, f}$ Keiichi Shimamura, ${ }^{b, f}$ Yuta Ujiie, ${ }^{b, g}$ Akari Arakawa,,f \\ Chie Kuroiwa, ${ }^{\text {b,f }}$ Mayuko Ishijima, ${ }^{\text {h }}$ Yuki Kishimoto, ${ }^{d}$ So Kanazawa, ${ }^{\text {b,i }}$ \\ Takanori Yamagata, ${ }^{c}$ Masami K. Yamaguchi, ${ }^{\text {b,j }}$ Ryoichi Sakuta,,f, \\ and Ippeita Dan ${ }^{\text {b,d }}$ \\ anstitute for Developmental Research, Aichi Developmental Disability Center, \\ Department of Functioning and Disability, Kagiya-cho, Kasugai, Aichi, Japan \\ ${ }^{b}$ RISTEX (Research Institute of Science and Technology for Society) Group, Kasuga, \\ Bunkyo, Tokyo, Japan \\ 'Jichi Medical University, Department of Pediatrics, Yakushiji, Shimotsuke, Tochigi, Japan \\ ${ }^{\mathrm{d}}$ Chuo University, Applied Cognitive Neuroscience Laboratory, Kasuga, Bunkyo, \\ Tokyo, Japan \\ ${ }^{\mathrm{e}}$ International University of Health and Welfare, Department of Pediatrics, Iguchi, \\ Nasushiobara, Tochigi, Japan \\ ${ }^{\mathrm{f}}$ Dokkyo Medical University, Child Development and Psychosomatic Medicine Center, \\ Minamikoshigaya, Koshigaya, Saitama, Japan \\ ${ }^{g}$ Chuo University, Research and Development Initiative, Kasuga, Bunkyo, Tokyo, Japan \\ ${ }^{h}$ Jichi Medical University, Yakushiji, Shimotsuke, Tochigi, Japan \\ iJapan Women's University, Department of Psychology, Nishi-Ikuta, Tama, Kawasaki, \\ Kanagawa, Japan \\ ${ }^{\mathrm{j} C h u o ~ U n i v e r s i t y, ~ D e p a r t m e n t ~ o f ~ P s y c h o l o g y, ~ H i g a s h i n a k a n o, ~ H a c h i o j i, ~ T o k y o, ~ J a p a n ~}$
}

\begin{abstract}
Significance: It has been reported that children with attention-deficit hyperactivity disorder (ADHD) have impairment in the recognition of angry but not of happy facial expressions, and they show atypical cortical activation patterns in response to facial expressions. However, little is known about neural mechanisms underlying the impaired recognition of facial expressions in school-aged children with ADHD and the effects of acute medication on their processing of facial expressions.
\end{abstract}

Aim: We aimed to investigate the possibility that acute administration of methylphenidate (MPH) affects processing of facial expressions in ADHD children.

Approach: We measured the hemodynamic changes in the bilateral temporo-occipital areas of ADHD children observing the happy and angry facial expressions before and $1.5 \mathrm{~h}$ after MPH or placebo administration in a randomized, double-blind, placebo-controlled, crossover design study.

Results: We found that, regardless of medication, happy expressions induced increased oxyhemoglobin (oxy-Hb) responses in the right inferior occipital region but not in the superior temporal region. For angry expressions, oxy-Hb responses increased after MPH administration, but not after placebo administration, in the left inferior occipital area, whereas there was no significant activation before MPH administration.

Conclusions: Our results suggest that (1) ADHD children consistently recruit the right inferior occipital regions to process happy expressions and (2) MPH administration to ADHD children enhances cortical activation in the left inferior occipital regions when they process angry expressions.

*Address all correspondence Megumi Kobayashi, E-mail: megumik@inst-hsc.jp 
(C) The Authors. Published by SPIE under a Creative Commons Attribution 4.0 Unported License. Distribution or reproduction of this work in whole or in part requires full attribution of the original publication, including its DOI. [DOI: 10.1117/1.NPh.7.2.025003]

Keywords: face processing; facial expressions; attention-deficit hyperactivity disorder; nearinfrared spectroscopy; occipito-temporal area.

Paper 19085R received Sep. 2, 2019; accepted for publication Apr. 16, 2020; published online May 6, 2020.

\section{Introduction}

Attention-deficit hyperactivity disorder (ADHD) is one of the most common psychiatric developmental disorders, and it affects $\sim 10 \%$ of school-aged children in the United States. ${ }^{1}$ ADHD is characterized by three core symptoms: inattention, hyperactivity, and impulsivity. Therefore, most research with ADHD children has focused on cognitive functions and its impairments related to these core symptoms (e.g., executive functions, attention, etc.). Recent findings, however, reveal that, in addition to these cognitive impairments, ADHD includes some deficits in social cognition.

One of the most important aspects of social cognition is emotion perception, especially through the processing of facial expressions. Faces convey a wealth of information about a person's emotional state, ${ }^{2}$ and we use them to successfully communicate socially with other individuals. Yet, it has been reported that $37 \%$ of school-aged ADHD children have impairment of emotion recognition. ${ }^{3}$ Atypical processing of facial expressions, especially negative expressions, such as anger, fear, and sadness, has been reported for children with $\mathrm{ADHD}^{4-14}$ and for schoolaged children at risk of ADHD, ${ }^{15}$ as well as for adult ADHD patients. ${ }^{16,17}$ Some previous studies revealed that ADHD children had poor performance in the recognition of angry facial expressions compared with typically developing (TD) children, whereas that of happy facial expressions was not significantly different between ADHD and TD children. ${ }^{5,8,13}$ In accordance with this behavioral evidence, neuroimaging studies with ADHD children have found atypical patterns of brain activation during recognition of facial expressions in the temporal areas, which are reported to be crucial for face processing. ${ }^{5,18}$ Williams et al. ${ }^{5}$ measured event-related potentials (ERPs) while ADHD children observed facial expressions and found that neural responses in ADHD children were significantly different from those in TD children for angry expressions but not for happy expressions. Furthermore, a near-infrared spectroscopic (NIRS) study compared hemodynamic responses with angry and happy expressions between ADHD children and TD children. ${ }^{18}$ ADHD children showed increased hemodynamic responses in the right temporal area for happy expressions but not for angry expressions, while TD children showed increased hemodynamic responses in the right temporal area for both facial expressions. ${ }^{18}$ Previous behavioral and neuroimaging findings suggest that the impaired ability to process facial expressions in ADHD children could be related to an atypical neural processing of facial expressions.

It has been reported that the behavioral and cognitive characteristics of ADHD are partly related to dopamine (DA) and noradrenaline (NA) dysfunctions ${ }^{19}$ and that impaired cognition and social functions improve with the administration of psychostimulant drugs, such as methylphenidate (MPH). ${ }^{20}$ For ADHD children, one of the most common first-choice treatments is the administration of MPH. It is believed that MPH increases synaptic transmission by inhibiting reuptake of catecholamines, mainly DA, and acts as a DA agonist in the cerebral cortices. ${ }^{21}$ Although it is known that MPH affects both the DA and NA systems, ${ }^{22,23}$ effects on the DA system are far greater than those on the NA system because MPH has an affinity to DA receptors 10 times higher than that to NA receptors. ${ }^{24}$ Recent studies have shown that MPH improves both cognitive performance and cerebral processing during cognitive tasks in ADHD children. ${ }^{25,26}$ For example, Monden et al. ${ }^{25}$ found that, although ADHD children had less accurate performance and lower activation in the right inferior and middle frontal gyri during a go/no-go task than did TD children before MPH administration, there were no differences in either accuracy or brain activity between ADHD and TD children after MPH administration.

Given that MPH improves neurocognitive processing during go/no-go tasks (e.g., Ref. 25), recognition of facial expressions might also be improved by MPH administration. One previous 
study investigated the effect of MPH on recognition of facial expressions and found that recognition performance for angry expressions 4 weeks after treatment with MPH was higher than that before treatment. ${ }^{5}$ Nonetheless, even after treatment with MPH, ADHD children's ability to recognize angry expressions was still significantly lower than that of TD children. This finding suggests that ADHD children's impaired recognition of facial expressions results from dysfunctions in the core cortical regions involved in the processing of facial expressions, such as the superior temporal sulcus (STS), as well as the fusiform gyrus (FG) and the inferior occipital gyrus (IOG), ${ }^{27}$ and that in ADHD children, an alternative or compensating processing of angry expressions may be driven as a result of MPH administration.

To investigate the possibility that acute administration of MPH affects processing of facial expressions in ADHD children, we used functional near-infrared spectroscopy (fNIRS) to measure cortical hemodynamic responses while ADHD children observed facial expressions in a randomized, double-blind, placebo-controlled, crossover design study. We compared hemodynamic responses in ADHD children's temporal and occipital areas during the presentation of angry and happy facial expressions both before and after MPH or a placebo administration. Considering previous evidence that MPH administration improves recognition of angry expressions, ${ }^{5}$ we predicted that cortical areas involved in the recognition of angry expressions might exhibit increased activation after MPH administration.

\section{Materials and Methods}

\subsection{Participants}

The final sample of participants for this study consisted of 19 clinically referred right-handed Japanese children (one female), who were diagnosed as ADHD based on the DSM- $5^{28}$ by trained pediatric neurologists and required administration of MPH (mean age $=9.84$ years, $\mathrm{SD}=$ 1.26 years, range 8 to 12 years; Table 1). An additional six ADHD boys participated but were excluded from the statistical analysis because of an insufficient number of viable trials due to failure to look at the face stimuli for more than three trials for either the happy or angry expression condition or due to motion artifacts. The full-scale IQ scores of participants were assessed using the Wechsler Intelligence Scale of Children Third (WISC-III) or Fourth (WISC-IV) and were all above 70 (mean $=92.68, \mathrm{SD}=14.0$, range 74 to 129). All participants had been taking MPH (18 to $45 \mathrm{mg} /$ day) as part of their regular medication regimen. Specific acute doses were the same as the patients' daily doses. The diagnosis of comorbid psychiatric conditions (e.g., autism spectrum disorder) was also established by experienced pediatric neurologists based on the DSM-5. ${ }^{28}$

All participants and their parents gave oral consent to participate in the study and written informed consent was obtained from the parents of all participants. The study was approved by the Ethics Committees of Jichi Medical University Hospital. The experiments were conducted in accordance with the latest version of the Declaration of Helsinki.

\subsection{Stimuli}

The same stimuli were used as in previous fNIRS studies. ${ }^{18,29}$ The stimuli consisted of full-color photographs of neutral, happy, and angry facial expressions of five Japanese females. The size of the face stimuli was $\sim 13 \times 10 \mathrm{deg}$ in visual angle. For the baseline, we presented a blank screen.

There were two test conditions: the happy expression condition and the angry expression condition. For both the happy and angry conditions, one of the five females was chosen randomly for each trial. The sequence of stimulus presentation was identical to that in the previous study. ${ }^{18}$ We presented an image of a neutral face for $400 \mathrm{~ms}$ followed by a happy or angry expressions for $400 \mathrm{~ms}$, which allowed participants to perceive the presented faces as having dynamic expressions that changed from neutral to happy or from neutral to angry. The 200-ms interstimulus interval was filled with a fixation point (black dot; $3.5 \times 3.5$ deg in visual angle). Each trial lasted $10 \mathrm{~s}$ and followed a baseline period of at least $20 \mathrm{~s}$. The duration of the baseline period was controlled by an experimenter. To draw and retain the children's attention, fixation 
Table 1 Demographic and clinical profiles

\begin{tabular}{|c|c|c|c|c|c|c|c|c|}
\hline ID & $\begin{array}{c}\text { Age } \\
\text { (years) }\end{array}$ & Sex & $\begin{array}{l}\text { ADHD } \\
\text { subtype }\end{array}$ & Complication & $\begin{array}{l}\text { MPH } \\
\text { dose } \\
(\mathrm{mg})\end{array}$ & $\begin{array}{l}\text { WISC } \\
\text { III or IV } \\
\text { Full-IQ }\end{array}$ & $\begin{array}{l}\text { Duration of } \\
\text { MPH exposure } \\
\text { (months) }\end{array}$ & Other medication \\
\hline 1 & 10 & Female & Combined & None & 45 & 74 & 2.8 & $\begin{array}{l}\text { Levocetirizine } \\
\text { hydrochloride, } \\
\text { magnesium oxide }\end{array}$ \\
\hline 2 & 12 & Male & Inattentive & None & 27 & 80 & 1.0 & None \\
\hline 3 & 9 & Male & Combined & None & 18 & 84 & 0.5 & None \\
\hline 4 & 12 & Male & Inattentive & None & 27 & 85 & 2.2 & None \\
\hline 5 & 10 & Male & Combined & None & 18 & 94 & 2.3 & None \\
\hline 6 & 8 & Male & Combined & None & 18 & 92 & 0.2 & None \\
\hline 7 & 11 & Male & Combined & ASD & 18 & 120 & 2.3 & Aripiprazole \\
\hline 8 & 8 & Male & Combined & ASD & 27 & 105 & 0.6 & Aripiprazole \\
\hline 9 & 11 & Male & Combined & None & 27 & 76 & 2.1 & None \\
\hline 10 & 10 & Male & Inattentive & ASD & 18 & 129 & 0.7 & None \\
\hline 11 & 10 & Male & Combined & None & 27 & 85 & 1.9 & Aripiprazole \\
\hline 12 & 11 & Male & Combined & None & 27 & 86 & 1.9 & None \\
\hline 13 & 8 & Male & Combined & ASD & 27 & 96 & 2.2 & None \\
\hline 14 & 10 & Male & Inattentive & $\begin{array}{c}\text { Type II diabetes, } \\
\text { morbid obesity, } \\
\text { hyperlipidemia }\end{array}$ & 45 & 97 & 2.6 & None \\
\hline 15 & 11 & Male & Combined & None & 27 & 82 & 2.6 & None \\
\hline 16 & 9 & Male & Combined & ASD & 18 & 99 & 1.5 & None \\
\hline 17 & 9 & Male & Combined & None & 18 & 85 & 3.4 & None \\
\hline 18 & 9 & Male & Combined & None & 27 & 99 & 1.6 & None \\
\hline 19 & 9 & Male & Combined & ASD & 18 & 93 & 2.0 & None \\
\hline Mean & 9.8 & & & & & 92.7 & & \\
\hline SD & 1.3 & & & & & 14.0 & & \\
\hline
\end{tabular}

points during both the baseline and the test were accompanied by respective beeping sounds presented at $1 \mathrm{~Hz}$.

\subsection{Procedure}

Each participant was tested while sitting in a chair and looking at a computer screen $\sim 40 \mathrm{~cm}$ away. Participants observed the face stimuli passively while their brain activity was measured. During measurements, we monitored the participants' behavior to evaluate the validity of each trial.

\subsection{Experimental Design}

The effects of MPH were examined in a randomized, double-blind, placebo-controlled, crossover study while participants observed happy and angry facial expressions (Fig. 1). 


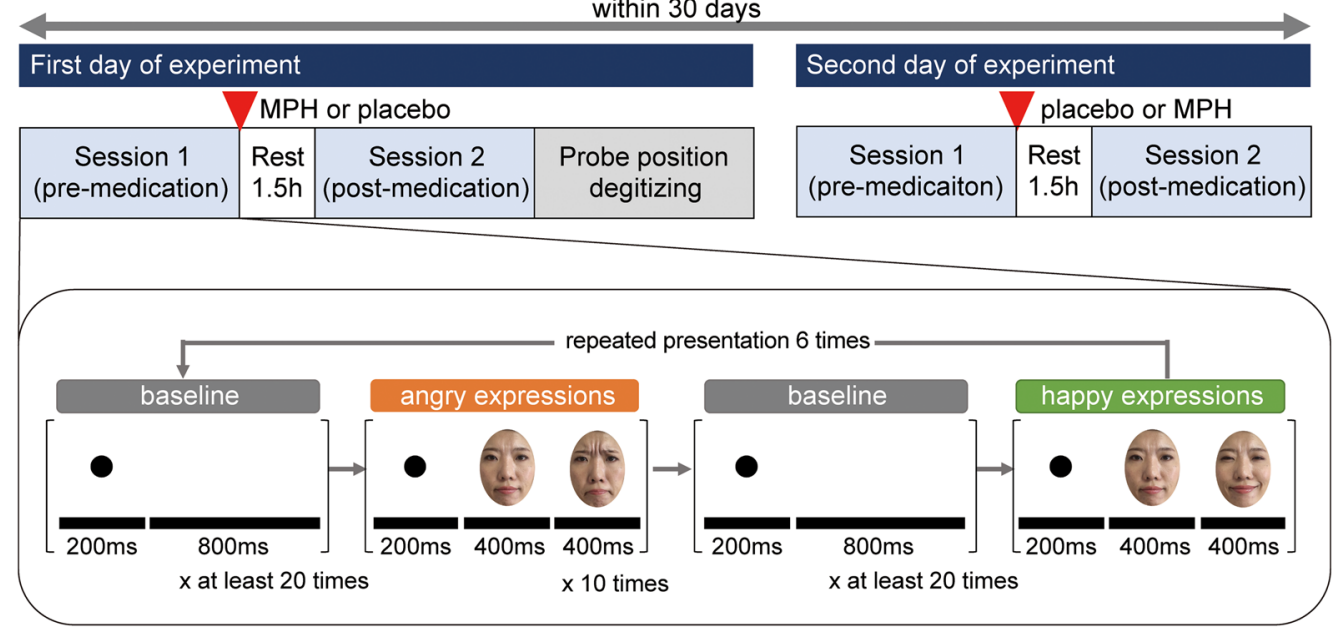

Fig. 1 Experimental design. A schematic showing the flow of a pre- and postmedication administration session.

We investigated ADHD participants twice within 30 days: one day for medication with MPH and the other day for medication with a placebo. The order of medication of MPH or a placebo was pseudorandomized across participants to avoid order effects.

On each day, ADHD participants underwent two sessions, one before administration of MPH or placebo and the other at $1.5 \mathrm{~h}$ after administration. Before each premedication session, all participants underwent an MPH washout period of 4 days. According to the previous study, ${ }^{18}$ each session consisted of 12 trials ( 6 trials for the happy expression condition and 6 for the angry expression condition). The order in which the two facial expressions were presented (happy expressions first or angry expressions first) was counterbalanced across participants and was the same across all four sessions for each participant.

After measurement of the first session, either MPH (OROS-MPH or Concerta) or a placebo was administered orally. Specific acute doses were the same as the participants' daily doses, as shown in Table 1 .

\section{5 fNIRS Measurements}

We used the multichannel fNIRS system ETG-4000 (Hitachi corporation, Tokyo, Japan) with two wavelengths of near-infrared light $(695$ and $830 \mathrm{~nm})$ and two sets of fNIRS probes $(3 \times 5$ array) to measure oxygenated hemoglobin (oxy-Hb) and deoxygenated hemoglobin (deoxy-Hb) signal changes from 44 channels with a sampling rate of $10 \mathrm{~Hz}$. We analyzed optical data based on the modified Beer-Lambert's law, ${ }^{30}$ as previously described. ${ }^{31} \mathrm{Oxy}-\mathrm{Hb}$ and deoxy-Hb signal changes were calculated in units of millimolar-millimeter (mM-mm). ${ }^{31,32}$

Each fNIRS probe contained 15 optical fibers with eight emitters and seven detectors, which were arranged alternately with an emitter-detector distance of $3 \mathrm{~cm}$. Each pair of adjacent emitting and detecting fibers was defined as a single measurement channel, resulting in 22 channels (Ch) for each probe.

We set the fNIRS probes at the bilateral temporal areas because these regions have been reported as important regions for face processing (e.g., Refs. 33 and 34). The probes in the present study covered the probe placements by Ichikawa et al. ${ }^{18}$ After positioning of the probes, the experimenter confirmed whether the fibers were touching each participant's scalp correctly. If inadequate contact between the fibers and the participant's scalp was detected, channels were excluded from statistical analysis.

After measurement, we obtained positional data for emitters, detectors, and reference points (nasion: Nz, midline central: $\mathrm{Cz}$, and left and right preauricular points) in real-world space using a 3D digitizer (Eastscan, Polhemus). To identify the spatial positions of measurement channels, we adopted the probabilistic registration method ${ }^{35-37}$ for registering fNIRS data to Montreal Neurological Institute (MNI) standard brain space, as described in previous studies (e.g., 
Kobayashi et al.: Acute administration of methylphenidate differentially affects cortical processing...

Table 2 Spatial profiles of the channels activated for facial expressions

\begin{tabular}{|c|c|c|c|c|c|c|}
\hline & & $\begin{array}{c}\text { MNI coordinates } \\
x, y, z \text { (SD) }\end{array}$ & Macroanatomy & Prob. & BA & Prob. \\
\hline \multirow[t]{3}{*}{ Left } & Channel 18 & $-52,-78,-10(14)$ & Left inferior occipital gyrus & 0.63 & $19-\mathrm{V} 3$ & 0.70 \\
\hline & & & Left middle occipital gyrus & 0.25 & $18-\mathrm{V} 2$ & 0.21 \\
\hline & & & & & 37-FA & 0.09 \\
\hline \multirow[t]{17}{*}{ Right } & Channel 4 & $58,-65,-22(14)$ & Cerebellum crus & 0.51 & $37-F A$ & 0.52 \\
\hline & & & Right inferior temporal gyrus & 0.40 & $19-\mathrm{V} 3$ & 0.38 \\
\hline & & & Right inferior occipital gyrus & 0.05 & $\begin{array}{l}20 \text {-Inferior temporal } \\
\text { gyrus }\end{array}$ & 0.11 \\
\hline & Channel 8 & $67,-55,-4(12)$ & Right middle temporal gyrus & 0.60 & $37-F A$ & 0.50 \\
\hline & & & Right inferior temporal gyrus & 0.40 & $\begin{array}{l}\text { 21-Middle temporal } \\
\text { gyrus }\end{array}$ & 0.49 \\
\hline & Channel 9 & $51,-80,-10(14)$ & Right inferior occipital gyrus & 0.58 & $19-\mathrm{V} 3$ & 0.61 \\
\hline & & & Right inferior temporal gyrus & 0.13 & $18-\mathrm{V} 2$ & 0.37 \\
\hline & & & Right middle occipital gyrus & 0.11 & & \\
\hline & Channel 10 & $62,17,23(14)$ & $\begin{array}{l}\text { Right inferior frontal } \\
\text { operculum }\end{array}$ & 0.37 & $\begin{array}{l}\text { 45-pars triangularis } \\
\text { Broca's area }\end{array}$ & 0.34 \\
\hline & & & $\begin{array}{l}\text { Right inferior frontal } \\
\text { triangularis }\end{array}$ & 0.29 & $\begin{array}{l}\text { 9-Dorsolateral } \\
\text { prefrontal cortex }\end{array}$ & 0.32 \\
\hline & & & Right precentral gyrus & 0.28 & $\begin{array}{l}\text { 44-Pars opercularis, } \\
\text { part of Broca's area }\end{array}$ & 0.23 \\
\hline & Channel 13 & $59,-70,7(14)$ & Right middle temporal gyrus & 0.72 & $19-\mathrm{V} 3$ & 0.41 \\
\hline & & & Right middle occipital gyrus & 0.16 & $37-F A$ & 0.24 \\
\hline & & & Right inferior temporal gyrus & 0.11 & $\begin{array}{l}\text { 39-Angular gyrus, } \\
\text { part of Wernicke's } \\
\text { area }\end{array}$ & 0.23 \\
\hline & Channel 15 & $66,-2,36(14)$ & Right postcentral gyrus & 0.63 & 43-Subcentral area & 0.40 \\
\hline & & & Right precentral gyrus & 0.37 & $\begin{array}{l}\text { 6-Premotor and } \\
\text { supplementary } \\
\text { motor cortex }\end{array}$ & 0.82 \\
\hline & & & & & $\begin{array}{l}\text { 9-Dorsolateral } \\
\text { prefrontal cortex }\end{array}$ & 0.12 \\
\hline
\end{tabular}

Note: Prob., probability that a given channel is located on an indicated cortical structure; SD, standard deviation.

Ref. 26). Spatial profiles of the channels where activation was observed during presentation of facial expressions are shown in Table 2.

\subsection{Data Analysis}

Before analyzing the data, we evaluated the validity of each trial. We excluded a trial from analysis if (a) participants did not look at the face stimuli or (b) body movements were detected based on the experimenter's visual examination during measurements. In the current study, no channels were rejected for either happy expression or angry expression conditions. 
We analyzed oxy-Hb and deoxy-Hb concentrations for further analysis. Individual timecourse data for the oxy- $\mathrm{Hb}$ and deoxy-Hb changes of each channel were preprocessed with a first-degree polynomial fitting and bandpass filter using cut-off frequencies of 0.01 to $0.8 \mathrm{~Hz}$ to remove baseline drift or noise from heartbeat pulsations. Based on the preprocessed time series data, channel-wise and participant-wise contrasts were obtained for each channel by calculating the intertrial mean of differences between the oxy-Hb or deoxy-Hb changes for tests (from 5 to $15 \mathrm{~s}$ after face stimulus onset) and baseline ( $3 \mathrm{~s}$ before face stimulus onset). We removed the trials if sudden, obvious, and sharp changes were detected in the time courses of oxy-Hb changes based on independent visual examination.

\subsection{Statistical Analysis}

We performed statistical analyses for the oxy-Hb and deoxy-Hb signals of each channel. We calculated the following contrasts: (1) averaged premedication contrast: averaged activation between preplacebo and pre-MPH versus baseline, (2) postmedication contrasts: face stimuli versus baseline activations for postplacebo and post-MPH, (3) intramedication contrast: difference between post- and premedication activations for each medication (i.e., "postplacebopreplacebo" and "post-MPH-pre-MPH"), and (4) intermedication contrast: difference between intra-MPH and intraplacebo contrasts (i.e., "post-MPH-pre-MPH" versus "postplacebopreplacebo"). For all contrasts, we performed two-tailed one-sample $t$-tests against zero with an effective multiplicity ( $M_{\text {eff }}$ ) correction method ${ }^{38}$ to correct familywise error. In $M_{\text {eff }}$ correction, fNIRS data were obtained from 44 channels (22 channels for each hemisphere) for 19 participants in each condition, and summary data for analyses were denoted as $\beta^{44 \times 19}$. Using the eigenvalues derived from a correlation matrix $(44 \times 44)$ of the measured signals $\left(\beta^{44 \times 19}\right)$, the $M_{\text {eff }}$ value was calculated for each contrast for each facial expression (angry and happy). The statistical significance level of 0.05 was divided by each $M_{\text {eff }}$ value.

\section{Results}

We obtained hemodynamic responses from 19 ADHD children who observed face stimuli for more than three trials for both happy and angry expression conditions. The mean number of trials was $5.05(\mathrm{SD}=0.91)$ for happy expressions and $4.95(\mathrm{SD}=1.13)$ for angry expressions before $\mathrm{MPH}$ administration, and $4.89(\mathrm{SD}=1.05)$ for happy expressions and $4.89(\mathrm{SD}=1.05)$ for angry expressions after MPH administration. Before placebo administration, the mean number of trials was $4.89(\mathrm{SD}=0.94)$ for happy expressions and $5.05(\mathrm{SD}=0.91)$ for angry expressions, and it was $5.05(\mathrm{SD}=0.85)$ for happy expressions and $5.21(\mathrm{SD}=0.92)$ for angry expressions after placebo administration. We conducted a three-way repeated-measures analysis of variance (ANOVA) on the number of trials with medication (MPH versus placebo), session (pre versus post), and condition (happy versus angry) as a within-subject factor and found no significant main effect or interaction. The mean number of valid trials in the current study was almost the same as that in the previous study. ${ }^{18}$

\subsection{Analyses of Oxy-Hb Signals}

Comparison of averaged oxy-Hb concentrations between preplacebo/pre-MPH and a baseline of zero (averaged premedication contrast) revealed that happy expressions induced significant increases of oxy- $\mathrm{Hb}$ concentration in the right channel $4(M=0.031, p<0.01$, Cohen's $d=0.69)$ and the right channel $8(M=0.025, p<0.01$, Cohen's $d=0.78)$, with an $M_{\text {eff }}$ value of 12.04 [Fig. 2(a) and Table 3]. For angry facial expressions, we found significant increases in the right channel $10(M=0.045, p<0.01$, Cohen's $d=0.81)$ and the right channel 15 $(M=0.041, p<0.01$, Cohen's $d=0.81)$, with an $M_{\text {eff }}$ value of 12.82 [Fig. 3(a) and Table 4].

After medication with MPH, oxy-Hb signal in the right channel 13 increased significantly compared with the baseline $(M=0.056, p<0.05$, Cohen's $d=0.68)$ for happy expressions, with an $M_{\text {eff }}$ value of 10.69 [Fig. 2(b) and Table 3], and oxy-Hb signal increased significantly in the left channel $18(M=0.051, p<0.05$, Cohen's $d=0.76)$ and the right channel 19 
(a) Pre-medication
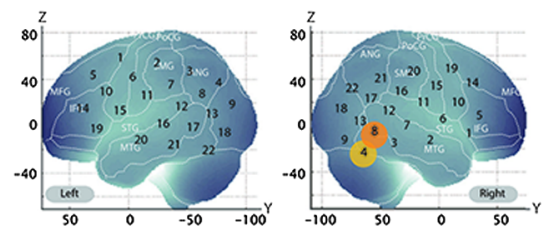

(b) Post-medication

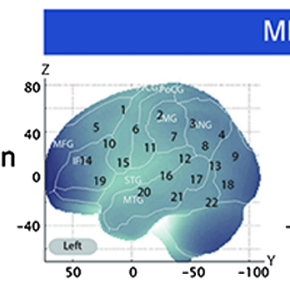

\section{MPH}

(c) Inter-medication
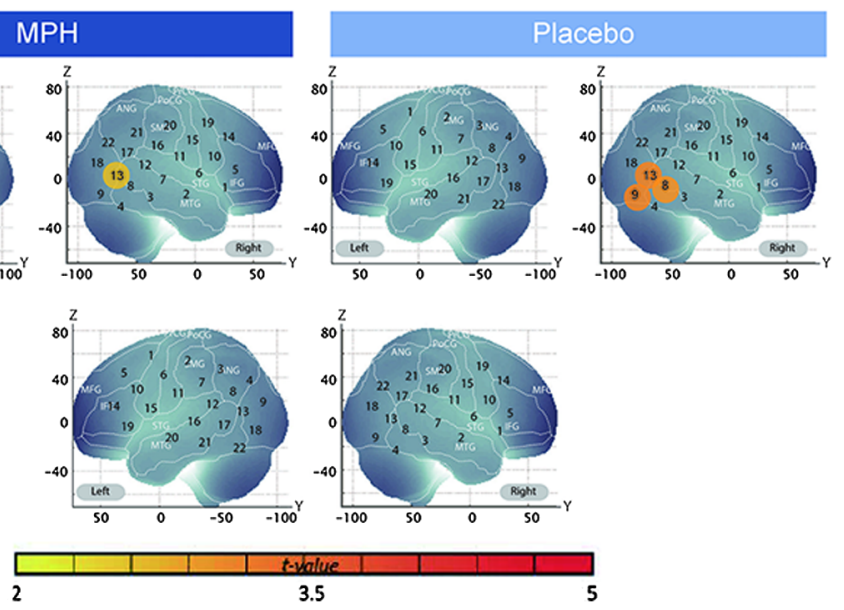

Fig. 2 Cortical hemodynamic responses for happy facial expressions before and after MPH and placebo administration. The t-maps of oxy- $\mathrm{Hb}$ concentration are shown with significant $t$-values (two-tailed one-sample $t$-test with $M_{\text {eff }}$ correction). All coordinates are in MNI space. (a) Premedication contrasts: averaged activation between preplacebo and pre-MPH versus baseline, (b) postmedication contrasts: face stimuli versus baseline for postplacebo and post-MPH, and (c) intermedication contrasts: difference between intra-MPH and intraplacebo contrasts ("post-MPH-pre-MPH" versus "post-placebo-preplacebo").

Table 3 Channels showing significant changes in oxy- $\mathrm{Hb}$ signals compared with baseline in contrast to happy facial expressions.

\begin{tabular}{llllll}
\hline \hline & & Mean & SD & $t$ & $p$ \\
\hline Premedication & Right channel 4 & 0.031 & 0.05 & 3.01 & $0.008^{*}$ \\
& Right channel 8 & 0.025 & 0.03 & 3.42 & $0.003^{*}$ \\
Post-MPH & Right channel 13 & 0.056 & 0.08 & 2.98 & $0.008^{*}$ \\
Postplacebo & Right channel 8 & 0.039 & 0.05 & 3.30 & $0.004^{*}$ \\
& Right channel 9 & 0.068 & 0.09 & 3.63 & $0.003^{*}$ \\
Intra-MPH & Right channel 13 & 0.058 & 0.08 & 3.38 & $0.003^{*}$ \\
Intraplacebo & - & & & & \\
Intermedication & - & & & & \\
\hline \hline
\end{tabular}

Note: SD, standard deviation; $t, t$-value; $p, p$ value.

${ }^{*}$ Statistically significant ( $p$ values are corrected by $M_{\text {eff }}$ correction).

( $M=0.046, p<0.05$, Cohen's $d=0.74$ ) for angry expressions, with an $M_{\text {eff }}$ value of 12.15 . [Fig. 3(b) and Table 4]. On the other hand, placebo administration induced significant oxy-Hb activations for happy expressions in the right channel $8(M=0.039, p<0.05$, Cohen's $d=0.76)$, the right channel $9(M=0.068, p<0.05$, Cohen's $d=0.77)$, and the right channel $13(M=0.058, p<0.05$, Cohen's $d=0.78)$, with an $M_{\text {eff }}$ value of 12.8 . However, no significant changes in oxy-Hb signal were observed for angry expressions, with an $M_{\text {eff }}$ value of 12.57 . 
(a) Pre-medication
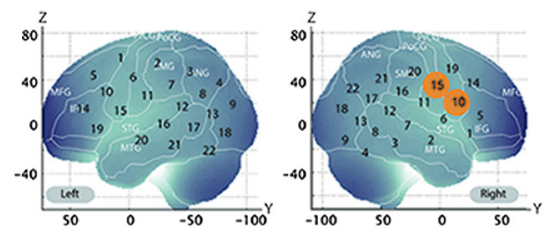

(b) Post-medication

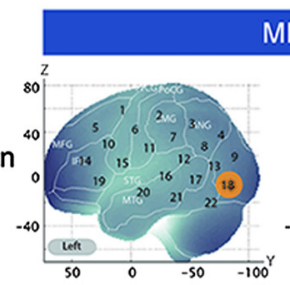

\section{$\mathrm{MPH}$}

(c) Inter-medication
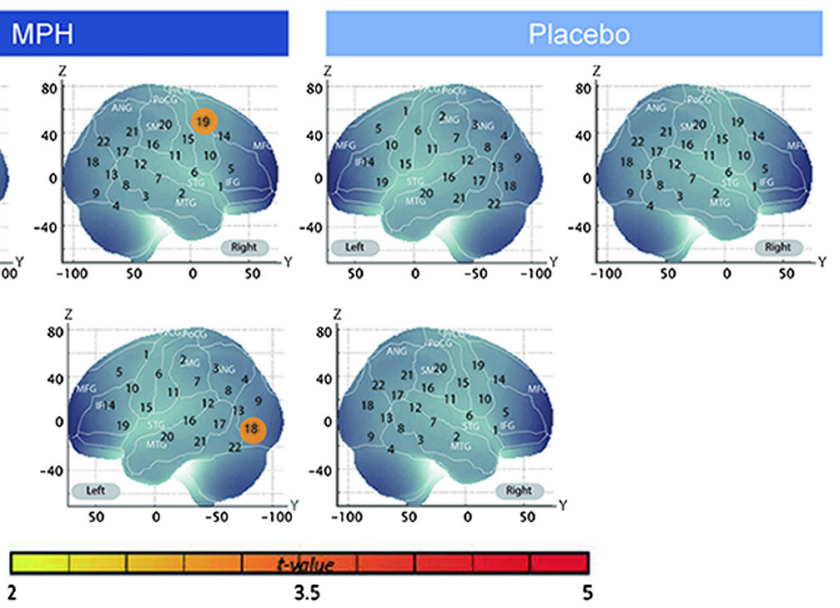

Fig. 3 Cortical hemodynamic responses for angry facial expressions before and after MPH and placebo administration. The t-maps of oxy- $\mathrm{Hb}$ concentration are shown with significant $t$-values (two-tailed one-sample $t$-test with $M_{\text {eff }}$ correction). All coordinates are in MNI space. (a) Premedication contrasts: averaged activation between preplacebo and pre-MPH versus baseline, (b) postmedication contrasts: face stimuli versus baseline for postplacebo and post-MPH, and (c) intermedication contrasts: difference between intra-MPH and intraplacebo contrasts ("post-MPH-pre-MPH" versus "postplacebo-preplacebo").

Table 4 Channels showing significant changes in oxy-Hb signals compared with baseline in contrast to angry facial expressions.

\begin{tabular}{llllll}
\hline \hline & & Mean & SD & $t$ & $p$ \\
\hline Premedication & Right channel 10 & 0.045 & 0.06 & 3.53 & $0.002^{*}$ \\
& Right channel 15 & 0.041 & 0.05 & 3.52 & $0.002^{*}$ \\
Post-MPH & Left channel 18 & 0.051 & 0.07 & 3.30 & $0.004^{*}$ \\
& Right channel 19 & 0.046 & 0.06 & 3.24 & $0.005^{*}$ \\
Postplacebo & - & & & \\
Intra-MPH & - & & & & \\
Intraplacebo & - & & & & \\
Intermedication & Left channel 18 & 0.058 & 0.08 & 3.28 & $0.004^{*}$ \\
\hline \hline
\end{tabular}

Note: SD, standard deviation; $t, t$-value; $p, p$ value.

*Statistically significant ( $p$ values are corrected by $M_{\text {eff }}$ correction).

To examine the effects of administering a placebo or MPH, we assessed the intramedication contrast, which is the difference between post- and premedication. We found that oxy-Hb signal after MPH administration was marginally higher than that before MPH administration only for angry faces in the left channel $18(M=0.044, p<0.10$, Cohen's $d=0.66)$, with an $M_{\text {eff }}$ value of 12.81. Conversely, there was no significant difference for either happy expressions, with an $M_{\text {eff }}$ value of 13.01 , or angry expressions, with an $M_{\text {eff }}$ value of 11.87 , with placebo administration. 
Finally, we analyzed the intermedication contrast to investigate any effect of medication with $\mathrm{MPH}$ on oxy-Hb changes during the presentation of angry expressions and happy expressions that was not present with the placebo. We found that the left channel 18 reached significance ( $M=0.058, p<0.05$, Cohen's $d=0.75$ ) for angry expressions, with an $M_{\text {eff }}$ value of 12.20 [Fig. 3(c) and Table 4], but not for happy expressions, with an $M_{\text {eff }}$ value of 12.96 [Fig. 2(c) and Table 3]. These results indicate that MPH, but not the placebo, activated the region around the left IOG when ADHD children observed angry faces.

\subsection{Analyses of Deoxy-Hb Signals}

Tables 5 and 6 represent the channels showing significant changes in deoxy-Hb signals. As for the averaged premedication contrast, which is a comparison of averaged signals between preplacebo/pre-MPH and the baseline of zero, we found no significant changes in any channels for both happy expressions, with an $M_{\text {eff }}$ value of 12.07 , and angry expressions, with an $M_{\text {eff }}$ value of 12.52 .

MPH administration induced significant decreases in deoxy-Hb signals for angry expressions in the right channel $15(M=-0.020, p<0.05$, Cohen's $d=0.82)$ and the right channel 19

Table 5 Channels showing significant changes in deoxy-Hb signals compared with baseline in contrast to happy facial expressions.

\begin{tabular}{|c|c|c|c|c|c|}
\hline & & Mean & SD & $t$ & $p$ \\
\hline Premedication & - & & & & \\
\hline Post-MPH & - & & & & \\
\hline Postplacebo & Left channel 18 & -0.022 & 0.02 & -3.78 & $0.001^{*}$ \\
\hline Intra-MPH & - & & & & \\
\hline \multirow[t]{3}{*}{ Intraplacebo } & Left channel 20 & -0.044 & 0.05 & -3.45 & $0.003^{*}$ \\
\hline & Right channel 3 & -0.020 & 0.02 & -3.89 & $0.001^{\star}$ \\
\hline & Right channel 17 & -0.032 & 0.03 & -4.07 & $0.001^{*}$ \\
\hline Intermedication & - & & & & \\
\hline
\end{tabular}

Note: SD, standard deviation; $t, t$-value; $p, p$ value.

*Statistically significant ( $p$ values are corrected by $M_{\text {eff }}$ correction).

Table 6 Channels showing significant changes in deoxy- $\mathrm{Hb}$ compared with baseline in contrast to angry facial expressions.

\begin{tabular}{llllll}
\hline \hline & & Mean & SD & $t$ & $p$ \\
\hline Premedication & - & & & \\
Post-MPH & Right channel 15 & -0.020 & 0.02 & -3.49 & $0.003^{*}$ \\
& Right channel 19 & -0.032 & 0.04 & -3.30 & $0.004^{*}$ \\
Postplacebo & - & & & \\
Intra-MPH & - & & & \\
Intraplacebo & - & & & \\
Intermedication & - & & & \\
\hline \hline
\end{tabular}

Note: SD, standard deviation; $t, t$-value; $p, p$ value.

*Statistically significant ( $p$ values are corrected by $M_{\text {eff }}$ correction). 
( $M=-0.032, p<0.05$, Cohen's $d=0.78$ ), with an $M_{\text {eff }}$ value of 12.44 . We found no significant changes for happy expressions in all channels, with an $M_{\text {eff }}$ value of 9.78. After placebo administration, deoxy-Hb concentrations were significantly decreased in the left channel 18 ( $M=-0.022, p<0.05$, Cohen's $d=0.89$ ) only for happy expressions, with an $M_{\text {eff }}$ value of 12.97, but not for angry expressions, with an $M_{\text {eff }}$ value of 13.59 .

For the intramedication contrast, there was no significant difference for both happy expressions, with an $M_{\text {eff }}$ value of 10.74 , and angry expressions, with an $M_{\text {eff }}$ value of 12.12 , with MPH administration. After placebo administration, we found significant decreases in deoxy- $\mathrm{Hb}$ signals in the left channel $20(M=-0.044, p<0.05$, Cohen's $d=0.81)$, the right channel 3 $(M=-0.020, p<0.05$, Cohen's $d=0.92)$, and the right channel $17(M=-0.032, p<0.05$, Cohen's $d=0.96$ ) for happy expressions, with an $M_{\text {eff }}$ value of 13.57. In contrast, no significant changes were found for angry expressions, with an $M_{\text {eff }}$ value of 13.03 .

For the intermedication contrast, no channels reached significance either for happy expressions, with an $M_{\text {eff }}$ value of 11.84 , or for angry expressions, with an $M_{\text {eff }}$ value of 12.16 .

\section{Discussion}

The current study explored whether administration of MPH affects cortical processing of facial expressions in school-aged ADHD children using fNIRS. To this end, we measured hemodynamic responses in temporal and occipital regions during the presentation of angry and happy facial expressions before and after medication with MPH or a placebo. We found that happy facial expressions induced significant increases in oxygenated hemodynamic (oxy-Hb) responses compared with baseline in the right inferior occipital area before and after administration of either a placebo or MPH. On the other hand, angry facial expressions induced no significant increases before MPH administration, whereas increased oxy-Hb responses occurred in the left inferior occipital area after MPH administration. This pattern of brain activation was not found for placebo administration. Importantly, we found that the MPH-induced activation was significantly higher than the placebo-induced activation in the left inferior occipital area in response to angry facial expressions but not to happy facial expressions.

We used the same stimuli and compatible procedures as a previous study that investigated the perception of angry and happy facial expressions in ADHD children, ${ }^{18}$ and our results are consistent with the previous findings. As for the activation with happy expressions, we found that the channels in the right inferior occipital region showed increasing oxy-Hb responses consistently across sessions. Although the left inferior occipital region showed weak oxy-Hb responses to happy expressions before $M_{\text {eff }}$ correction, they did not reach statistical significance. These patterns of brain activation are concordant with the previous findings, ${ }^{18}$ showing a significant increase for happy expressions in the right temporal area but not in the left temporal area (note that channels covering temporal and occipital areas in each hemisphere were investigated in the previous study). For angry expressions, we revealed no significant increases of oxy-Hb signals in any channel except after MPH administration, which is also consistent with the previous study. ${ }^{18}$ Taken together, our results replicated the previous findings reported by Ichikawa et al. ${ }^{18}$

The results showing a significant activation for happy expressions regardless of medication are consistent with previous behavioral findings that ADHD children show comparable recognition performance for happy expressions relative to TD children. ${ }^{5,8,13}$ Also, our finding of increased hemodynamic responses in the right hemisphere, but not in the left, is in line with evidence of right hemispheric advantage for processing of facial expressions in adults ${ }^{39-42}$ and, thus, supports the notion that ADHD children have a preserved ability to process happy facial expressions.

The results we obtained from the activation levels of each channel may provide new insights into the neural processing of facial expressions in ADHD children. In the current study, ADHD children showed significant increases of oxy-Hb signal for happy facial expressions at the channels located in the inferior occipital region of the right hemisphere (Brodmann area (BA) 19: right IOG rather than the superior temporal region. Considering a former study showing that the face area at the IOG [occipital face area (OFA)] is located in BA 18 or $19,{ }^{43}$ the increased oxy-Hb signal for happy facial expressions may reflect face-specific processing in the right inferior 
occipital area or on a pathway from the IOG to the STS. It has been reported that face-specific activations are typically observed in the IOG (OFA), the fusiform gyrus (FG) [fusiform face area (FFA)], and the posterior superior temporal sulcus (pSTS face area). ${ }^{27}$ The neural model of face processing proposes that there are two distinct pathways for processing faces: the dorsal pathway (from the OFA to the pSTS) is involved in processing changeable facial information (e.g., facial expressions, eye gaze, and head rotation), whereas the ventral pathway (from the OFA to the FFA) is involved in processing invariant facial information (e.g., identity, race, and age). ${ }^{34,44}$ Consistent with this neural model, one fMRI study with adults revealed that there was a subnetwork for face processing that includes the left and right STS, which are involved in processing facial expressions, and that the bilateral OFAs play an important role in the face processing. Participants exhibited a significant decrease in functional connectivity between the bilateral OFAs and the right STS when they switched from a face recognition task to an object recognition task. ${ }^{45}$ Additionally, the OFA acts as the first node in this expression subnetwork and projects information about physical form or face parts to the STS, ${ }^{43,46-49}$ whereas the pSTS is sensitive to faces which convey valence information. ${ }^{50}$ Given these previous findings and considering the activation pattern exhibiting the inferior occipital region but not superior temporal regions, although ADHD children's recognition of happy expressions is thought to be intact, ${ }^{5,8,13}$ our results suggest that they may actually be relying more heavily on the cortical processing of physical form or facial parts than on the valence conveyed by facial expressions when observing the happy expressions.

A previous finding showing a strong functional connectivity between the bilateral OFAs and the right $\mathrm{STS}^{45}$ may also give an account of the significant MPH-induced activation in the left inferior occipital region (left BA 19) while observing angry facial expressions. In contrast to our prediction that MPH administration would induce activation in the right temporal area when observing angry expressions, which was based on previous findings showing a significant activation in the right temporal area in TD children, ${ }^{18}$ we found that MPH, but not a placebo, induced increases of oxy-Hb concentrations in the left inferior occipital area. Our results, however, are consistent with a previous ERP study that reported a significant improvement in the amplitude of the P300 component with MPH around the left posterior temporal region with angry expressions. ${ }^{5}$ One possibility may be that MPH could lead to the promotion of processing in the left occipital inferior region, which is part of the dorsal pathway related to facial expression processing. As mentioned above, both the left and right OFAs have a strong functional connectivity with the right STS. ${ }^{45}$ This suggests that the bilateral OFAs convey physical form information of faces to the right STS for processing of facial expressions. The MPH-induced activation in the left inferior occipital region observed in the current study may reflect that acute MPH administration promotes the processing of the physical form of angry expressions.

The significant increase in oxy-Hb signals with angry expressions induced by MPH administration is probably related to acute modulation of the DA system. Previous studies have shown that the DA system is important for recognition of angry expressions. ${ }^{51,52}$ For example, a selective disruption in the recognition of angry facial expressions, but not in other facial expressions, occurred in healthy adult participants with acute administration of a DA D2-class receptor antagonist. ${ }^{51}$ This finding implies that, although it is considered that the behavioral and cognitive characteristics of ADHD patients result partly from both DA and NA dysfunctions, ${ }^{19}$ an impairment in the recognition of angry faces in ADHD may be due to the DA dysfunction alone. Given that MPH acts as a DA agonist by blocking DA transporters, ${ }^{21}$ this MPH action could have induced increased oxy-Hb signals when ADHD children observed the angry faces.

In the present study, we found increased oxy-Hb responses in the region around the dorsolateral prefrontal cortex (DLPFC) and premotor cortex only for angry expressions before medication. This pattern of activation is consistent with previous findings that ADHD children exhibit greater activation in the DLPFC for angry expressions compared with TD children ${ }^{53}$ and may be related to emotional impairments in ADHD children. It has been reported that the DLPFC plays an important role in the modulation of aggressive behavior ${ }^{54}$ and regulation of emotional response. ${ }^{55,56}$ The premotor cortex is activated for perception of whole-body expressions of emotion ${ }^{57}$ and is involved in action preparation and execution. ${ }^{58,59}$ In addition, a previous study implied that activation in the premotor cortex when observing angry expressions may reflect autonomic reactions and motor responses related to defensive behaviors. ${ }^{60}$ Considering these 
previous findings, the increases in hemodynamic responses for angry expressions in the prefrontal and premotor areas may be related to evidence that ADHD children have more emotional impulsiveness, defined by quickness to anger, easy emotional excitability, low frustration tolerance, etc., than do control children. ${ }^{61}$

We found that, during passive observation of happy expressions, a slightly broader area of the right inferior occipital region activated after placebo administration (right channels 8,9 , and 13) compared with before placebo administration (right channels 4 and 8 ) in oxy-Hb signals. This pattern was also found in deoxy-Hb signals: no channel activated before placebo administration, while the left channel 18 activated after placebo administration. Previous EEG studies with psychostimulants ${ }^{62}$ and antidepressants ${ }^{63}$ reported a placebo effect in $\sim 30 \%$ of patients. Also, a placebo effect is occasionally observed within series of studies using the same double-blind, placebo-controlled, crossover-design experiments. ${ }^{25,64,65}$ In this respect, our results showing a broader activation after placebo administration may be partly due to a placebo effect, although the neural mechanisms behind this observation are still under debate. ${ }^{66}$

As opposed to oxy-Hb signals, we did not find significant changes in deoxy-Hb signals even for happy expressions in occipital temporal areas across sessions after multiplicity correction (some channels showed significant decreases before multiple correction). These results suggest that changes in deoxy-Hb signals would be a less sensitive indicator of cortical processing of facial expressions than changes in oxy-Hb signals. Our results are consistent with previous findings that the signal amplitude of oxy-Hb is higher than that of deoxy- $\mathrm{Hb}^{67}$ and that oxy- $\mathrm{Hb}$ is more sensitive to activation related to the processing of facial expressions. ${ }^{18,29}$ Also, Hoshi and Tanji ${ }^{68}$ mentioned that oxy-Hb is the most sensitive indicator of changes in regional brain activation. Alternatively, deoxy-Hb signals are known to often exhibit delayed responses compared with oxy-Hb signals, ${ }^{69}$ which might not have been well detected in the current experimental design. Considering these findings, our results imply that oxy-Hb signals are more appropriate as an index to evaluate cortical processing of facial expressions than deoxy-Hb signals.

With the exception of the right channel 19 for the angry expressions in a comparison between pre-MPH and post-MPH administration, most channels showing significant increases in oxy-Hb signals did not show significant decreases in deoxy-Hb signals. Although increases in oxy- $\mathrm{Hb}$ signals are considered to be accompanied by decreases in deoxy-Hb signals with regional brain activation, deoxy-Hb signals do not necessarily show this pattern of changes. ${ }^{68}$ For example, an increase or absence of changes in deoxy-Hb signals was also observed with increases in oxy$\mathrm{Hb}^{70-72}$ Thus, the pattern of changes in deoxy-Hb signals and the applicability of the deoxy-Hb parameter should be carefully evaluated and discussed in further exploration.

We will also discuss a few limitations of the current study. First, we could not provide evidence for how different regions of bilateral temporal areas contribute to the processing of facial expressions in TD children because we did not measure the brain activity of TD children for comparison. Second, we included ADHD children with and without comorbid autism spectrum disorders (ASD). In this study, participants included 13 ADHD children without comorbid ASD and 6 ADHD children with it. A recent fNIRS study implies that ADHD with ASD is characterized by a different neurofunctional pathology than that without ASD. ${ }^{26}$ Given this finding, our sampling may have passively brought a contamination effect from the comorbidity of ASD on the present results.

To investigate the possible effect of ASD comorbidity, supplemental analyses were performed on the oxy-Hb data of ADHD children without comorbid ASD $(n=13)$. We found almost the same tendency as with the full participant set, namely, significant increases in oxy-Hb signals only for angry expressions after MPH administration in the left inferior occipital area (left channel 18) $(M=0.058, p<0.05$, Cohen's $d=0.99)$, with an $M_{\text {eff }}$ value of 9.43 . Importantly, the tendency of a medication effect induced not by a placebo but by MPH was also observed in the left inferior occipital area only for the presentation of angry expressions: MPH-induced activation was significantly higher than placebo-induced activation before correction for multiple comparisons $(M=0.048, p=0.04)$, while it did not reach significance after $M_{\text {eff }}$ correction with a value of 9.32. Similarly, in the other contrasts, we found a tendency of oxy-Hb increases in the channels showing significant increases in the full participant set. Thus, we consider that the any contamination effect would be small in the current study. However, these points can be tested with future research to extend the current findings. 


\section{Conclusions}

In conclusion, the current fNIRS study investigating the possibility that acute administration of MPH affects the cortical processing of facial expressions in ADHD children revealed different modulations in cortical activation patterns in response to the presentation of angry and happy expressions before and after MPH administration. We revealed that ADHD children show increased activation in the right inferior occipital region for happy expressions regardless of MPH or a placebo administration. In contrast, for angry expressions, significant activation was found in the left inferior occipital area after MPH administration but not before MPH administration. Importantly, we found significant MPH-induced, but not placebo-induced, increases in hemodynamic response for angry expressions in the left inferior occipital region, suggesting that MPH promotes the processing of physical forms or facial parts, but not valence information, of angry facial expressions.

\section{Disclosures}

The authors declare that the research was conducted in the absence of any commercial or financial relationships that could be construed as a potential conflict of interest.

\section{Acknowledgments}

We appreciate the members of the RISTEX group for their assistance. This work was supported in part by JST-RISTEX to ID and the Grant-in-Aid for Scientific Research from the Japan Society for Promotion of Science to ID (Grant No. 17H05959) and MK (Grant No. 19K14492). Also, this research was supported in part by the Grant-in-Aid for Scientific Research on Innovative Areas "Construction of the Face-Body Studies in Transcultural Conditions" (Grant No. 17H06343).

\section{References}

1. L. J. Akinbami et al., "Attention deficit hyperactivity disorder among children aged 5-17 years in the United States, 1998-2009," NCHS Data Brief 70, 1-8 (2011).

2. R. D. Odom and C. M. Lemond, "Developmental differences in the perception and production of facial expressions," Child Dev. 43(2), 359-369 (1972).

3. D. Sjöwall et al., "Multiple deficits in ADHD: executive dysfunction, delay aversion, reaction time variability, and emotional deficits," J. Child Psychol. Psychiatry 54(6), 619-627 (2013).

4. D. P. Dickstein and F. X. Castellanos, "Face processing in attention deficit/hyperactivity disorder," in Behavioral Neuroscience of Attention Deficit Hyperactivity Disorder and its Treatment, Current Topics in Behavioural Neuroscience, Vol. 9, C. Stanford and R. Tannock, Eds., pp. 219-237, Springer, Heidelberg, Germany, eBook (2011).

5. L. M. Williams et al., "Misinterpreting emotional expressions in attention-deficit/ hyperactivity disorder: evidence for a neural marker and stimulant effects," Biol. Psychiatry 63, 917-926 (2008).

6. B. Corbett and H. Glidden, "Processing affective stimuli in children with attention-deficit hyperactivity disorder," Child Neuropsychol. 6(2), 144-155 (2000).

7. E. Demirci and A. Erdogan, "Is emotion recognition the only problem in ADHD? Effects of pharmacotherapy on face and emotion recognition in children with ADHD," ADHD Attention Deficit Hyperactivity Disorders 8(4), 197-204 (2016).

8. K. Pelc et al., "Recognition of emotional facial expressions in attention-deficit hyperactivity disorder," Pediatric Neurol. 35(2), 93-97 (2006).

9. D. Da Fonseca et al., "Emotion understanding in children with ADHD," Child Psychiatry Human Dev. 40, 111-121 (2009).

10. K. E. Seymour et al., "Emotional face identification in youths with primary bipolar disorder or primary attention-deficit/hyperactivity disorder," J. Am. Acad. Child Adolesc. Psychiatry 52(5), 537-546.e3 (2013). 
11. J. Sinzig, D. Morsch, and G. Lehmkuhl, "Do hyperactivity, impulsivity and inattention have an impact on the ability of facial affect recognition in children with autism and ADHD?" Eur. Child Adolesc. Psychiatry 17(2), 63-72 (2008).

12. N. Yuill and J. Lyon, "Selective difficulty in recognizing facial expressions of emotion in boys with ADHD," Eur. Child Adolesc. Psychiatry 16, 398-404 (2007).

13. S. D. Singh et al., "Recognition of facial expressions of emotion by children with attentiondeficit hyperactivity disorder," Behav. Modif. 22(2), 128-142 (1998).

14. K. Borhani and V. Nejati, "Emotional face recognition in individuals with attention-deficit/ hyperactivity disorder: a review article," Dev. Neuropsychol. 43(3), 256-277 (2018).

15. I. Kats-Gold, A. Besser, and B. Priel, "The role of simple emotion recognition skills among school aged boys at risk of ADHD," J. Abnormal Child Psychol. 35(3), 363-378 (2007).

16. E. Bora and C. Pantelis, "Meta-analysis of social cognition in attention-deficit/hyperactivity disorder (ADHD): comparison with healthy controls and autistic spectrum disorder," Psychol. Med. 46(4), 699-716 (2016).

17. M. Miller et al., "Affect recognition in adults with ADHD," J. Attention Disorders 15(6), 452-460 (2011).

18. H. Ichikawa et al., "Hemodynamic response of children with attention-deficit and hyperactivity disorder (ADHD) to emotional facial expressions," Neuropsychologia 63, 51-58 (2014).

19. T. Wilens, "Effect of methylphenidate on the catecholaminergic system in attention-deficit/ hyperactivity disorder," J. Clin. Psychopharmacol. 28(3) S46-S53 (2008).

20. T. J. Spencer, "ADHD treatment across the life cycle," J. Clin. Psychiatry 65, 22-26 (2004).

21. A. F. T. Arnsten, "Stimulants: therapeutic actions in ADHD," Neuropsychopharmacology 31, 2376-2383 (2006).

22. A. F. T. Arnsten and B. M. Li, "Neurobiology of executive functions: catecholamine influences on prefrontal cortical functions," Biol. Psychiatry 57(11), 1377-1384 (2005).

23. N. D. Volkow et al., "Activation of orbital and medial prefrontal cortex by methylphenidate in cocaine-addicted subjects but not in controls: relevance to addiction," J. Neurosci. 25(15), 3932-3939 (2005).

24. F. P. Bymaster et al., "Atomoxetine increases extracellular levels of norepinephrine and dopamine in prefrontal cortex of rat: a potential mechanism for efficacy in attention deficit/ hyperactivity disorder," Neuropsychopharmacology 27(5), 699-711 (2002).

25. Y. Monden et al., "Right prefrontal activation as a neuro-functional biomarker for monitoring acute effects of methylphenidate in ADHD children: an fNIRS study," NeuroImage Clin. 1, 131-140 (2012).

26. T. Tokuda et al., "Methylphenidate-elicited distinct neuropharmacological activation patterns between medication-naïve attention deficit hyperactivity disorder children with and without comorbid autism spectrum disorder: a functional near-infrared spectroscopy study," Neuropsychiatry 8(3), 917-929 (2018).

27. M. Bernstein and G. Yovel, "Two neural pathways of face processing: a critical evaluation of current models," Neurosci. Biobehav. Rev. 55, 536-546 (2015).

28. American Psychiatric Association, Diagnostic and Statistical Manual of Mental Disorders: DSM-5, American Psychiatric Publishing, Washington, DC (2013).

29. E. Nakato et al., "Distinct differences in the pattern of hemodynamic response to happy and angry facial expressions in infants-a near-infrared spectroscopic study," NeuroImage 54, 1600-1606 (2011).

30. M. Cope et al., "Methods of quantitating cerebral near infrared spectroscopy data," in Oxygen Transport to Tissue X, Advances in Experimental Medicine and Biology, M. Mochizuki et al., Eds., Vol. 215, pp. 183-189, Springer, Boston, Massachusetts (1988).

31. A. Maki et al., "Spatial and temporal analysis of human motor activity using noninvasive NIR topography," Med. Phys. 22(12), 1997-2005 (1995).

32. D. A. Shattuck et al., "Construction of a 3D probabilistic atlas of human cortical structures," NeuroImage 39(3), 1064-1080 (2008).

33. N. Kanwisher, J. McDermott, and M. M. Chun, "The fusiform face area: a module in human extrastriate cortex specialized for face perception," J. Neurosci. 17(11), 4302-4311 (1997). 
34. J. V. Haxby, E. A. Hoffman, and M. I. Gobbini, "The distributed human neural system for face perception," Trends Cognit. Sci. 4(6), 223-233 (2000).

35. D. Tsuzuki et al., "Stable and convenient spatial registration of stand-alone NIRS data through anchor-based probabilistic registration," Neurosci. Res. 72(2), 163-171 (2012).

36. D. Tsuzuki and I. Dan, "Spatial registration for functional near-infrared spectroscopy: from channel position on the scalp to cortical location in individual and group analyses," NeuroImage 85, 92-103 (2014).

37. D. Tsuzuki et al., "Virtual spatial registration of stand-alone fNIRS data to MNI space," NeuroImage 34(4), 1506-1518 (2007).

38. M. Uga et al., "Exploring effective multiplicity in multichannel functional near-infrared spectroscopy using eigenvalues of correlation matrices," Neurophotonics 2(1), 015002 (2015).

39. N. L. Etcoff, "Selective attention to facial identity and facial emotion," Neuropsychologia 22(3), 281-295 (1984).

40. K. Nakamura et al., "Activation of the right inferior frontal cortex during assessment of facial emotion," J. Neurophysiol. 82(3), 1610-1614 (1999).

41. N. Tsuchiya et al., "Decoding face information in time, frequency and space from direct intracranial recordings of the human brain," PLoS One 3(12), e3892 (2008).

42. G. Gainotti, "Unconscious processing of emotions and the right hemisphere," Neuropsychologia 50(2), 205-218 (2012).

43. D. Pitcher, V. Walsh, and B. Duchaine, "The role of the occipital face area in the cortical face perception network," Exp. Brain Res. 209, 481-493 (2011).

44. M. I. Gobbini and J. V. Haxby, "Neural systems for recognition of familiar faces," Neuropsychologia 45, 32-41 (2007).

45. Z. Zhen, H. Fang, and J. Liu, "The hierarchical brain network for face recognition," PLoS One 8(3), e59886 (2013).

46. P. Rotshtein et al., "Morphing Marilyn into Maggie dissociates physical and identity face representations in the brain," Nat. Neurosci. 8, 107-113 (2005).

47. J. Liu, A. Harris, and N. Kanwisher, "Perception of face parts and face configurations: an fMRI study," J. Cognit. Neurosci. 22, 203-211 (2010).

48. D. F. Nichols, L. R. Betts, and H. R. Wilson, "Decoding of faces and face components in face-sensitive human visual cortex," Front. Psychol. 1, 28 (2010).

49. D. Pitcher et al., "TMS evidence for the involvement of the right occipital face area in early face processing," Curr. Biol. 17, 1568-1573 (2007).

50. C. P. Said, J. V. Haxby, and A. Todorov, "Brain systems for assessing the affective value of faces," Philos. Trans. R. Soc. B 366, 1660-1670 (2011).

51. A. D. Lawrence et al., "Selective disruption of the recognition of facial expressions of anger," Neuroreport 13, 881-884 (2002).

52. A. J. Calder et al., "Impaired recognition of anger following damage to the ventral striatum," Brain 127, 1958-1969 (2004).

53. A. M. Passarotti, J. A. Sweeney, and M. N. Pavuluri, "Emotion processing influences working memory circuits in pediatric bipolar disorder and attention deficit hyperactivity disorder," J. Am. Acad. Child Adolesc. Psychiatry 49(10), 1064-1080 (2010).

54. P. R. Giancola, "Evidence for dorsolateral and orbital prefrontal cortical involvement in the expression of aggressive behavior," Aggressive Behav. 21, 431-450 (1995).

55. J. T. Buhle et al., "Cognitive reappraisal of emotion: a meta-analysis of human neuroimaging studies," Cereb. Cortex 24, 2981-2990 (2014).

56. M. L. Phillips et al., "Neurobiology of emotion perception I: the neural basis of normal emotion perception," Biol. Psychiatry 54, 504-514 (2003).

57. J. Dubois et al., "Causal mapping of emotion networks in the human brain: framework and initial findings," Neuropsychologia (in press).

58. N. Picard and P. L. Strick, "Imaging the premotor areas," Curr. Opin. Neurobiol. 11(6), 663-672 (2001).

59. E. Hoshi and J. Tanji, "Distinctions between dorsal and ventral premotor areas: anatomical connectivity and functional properties," Curr. Opin. Neurobiol. 17(2), 234-242 (2007). 
60. S. Pichon, B. de Gerlder, and J. Grèzes, "Emotional modulation of visual and motor areas by dynamic body expressions of anger," Social Neurosci. 3(3-4), 199-212 (2008).

61. R. A. Barkley and M. Fischer, "The unique contribution of emotional impulsiveness to impairment in major life activities in hyperactive children as adults," J. Am. Acad. Child Adolesc. Psychiatry 49(5), 503-513 (2010).

62. B. Saletu, P. Anderer, and G. M. Saletu-Zyhlarz, "EEG topography and tomography (LORETA) in the classification and evaluation of the pharmacodynamics of psychotropic drugs," Clin. EEG Neurosci. 37(2), 66-80 (2006).

63. H. H. Stassen et al., "Is there a common resilience mechanism underlying antidepressant drug response? Evidence from 2848 patients," J. Clin. Psychiatry 68(8), 1195-1205 (2007).

64. M. Nagashima et al., "Neuropharmacological effect of atomoxetine on attention network in children with attention deficit hyperactivity disorder during oddball paradigms as assessed using functional near-infrared spectroscopy," Neurophotonics 1(2), 025007 (2014).

65. M. Nagashima et al., "Acute neuropharmacological effects of atomoxetine on inhibitory control in ADHD children: a fNIRS study," Neurolmage Clin. 6, 192-201 (2014).

66. S. Geuter, L. Koban, and T. D. Wager, "The cognitive neuroscience of placebo effects: concepts, predictions, and physiology," Аnпи. Rev. Neurosci. 40, 167-188 (2017).

67. G. Strangman, D. A. Boas, and J. P. Sutton, "Non-invasive neuroimaging using nearinfrared light," Biol. Psychiatry 52, 679-693 (2002).

68. Y. Hoshi, "Chapter 7-Hemodynamic signals in fNIRS," Prog. Brain Res. 225, 153-179 (2016).

69. M. Uga et al., "Optimizing the general linear model for functional near-infrared spectroscopy: an adaptive hemodynamic response function approach," Neurophotonics 1(1), 015004 (2014).

70. Y. Hoshi and M. Tamura, "Dynamic multichannel near-infrared optical imaging of human brain activity," J. Appl. Physiol. 75, 1842-1846 (1993).

71. T. Kato et al., "Human visual cortical function during photic stimulation monitoring by means of near-infrared spectroscopy," J. Cereb. Blood Flow Metab. 13, 516-520 (1993).

72. A. Kleinschmidt et al., "Simultaneous recording of cerebral blood oxygenation changes during human brain activation by magnetic resonance imaging and near-infrared spectroscopy," J. Cereb. Blood Flow Metab. 16, 817-826 (1996).

Megumi Kobayashi received her PhD from Chuo University, Japan in 2013. She was a JSPS Research Fellow in Chuo University (2010-2013) and the National Institute for Physiological Sciences (NIPS) (2013-2016). After a research fellowship in NIPS (2016-2017), she has worked at the Institute for Developmental Research, Aichi Developmental Disability Center, as an assistant professor since 2017. Her research focuses on development of face and body perception/recognition in infants and children by behavioral and fNIRS methods.

Takahiro Ikeda received his medical degree in Japan in 2007 and his $\mathrm{PhD}$ from Jichi Medical University, Japan, in 2019. He was a resident from 2007 to 2012 and has been a research associate in the Department of Pediatrics, Jichi Medical University, Japan, since 2012. He was also a part of the medical staff at the International University of Health and Welfare Rehabilitation Center, Nasu Institute for Developmental Disabilities, Japan, from 2012 to 2014. His research focuses on neuroimaging studies of neurodevelopmental disorders using fNIRS.

Tatsuya Tokuda was a master's student at Chuo University, Japan. He engaged in fNIRS research from 2015 to 2020 . He has authored seven peer-reviewed articles. His research focuses on clinical applications of fNIRS.

Yukifumi Monden received his medical degree in Japan in 2002 and his PhD from Jichi Medical University, Japan, in 2013. His major professional achievements include being the chief intern at the U.S. Naval Hospital, Okinawa, Japan, from 2006 to 2007 and a clinical associate from 2009 to 2012. He is currently an assistant professor in the Department of Pediatrics at Jichi Medical University and has been a professor in the Department of Pediatrics, International University of Health and Welfare, Japan (since 2018). His research focuses on neuroimaging studies of neurodevelopmental disorders. 
Masako Nagashima received her medical degree in Japan in 2004. She was a resident from 2004 to 2009 and has been a research associate in the Department of Pediatrics, Jichi Medical University, Japan, since 2009. She was also a chief of inpatient medicine at the International University of Health and Welfare, Japan, from 2012 to 2013. She was a guest researcher at the University of Tübingen, Germany, from 2017 to 2019. Her research focuses on neuroimaging studies of neurodevelopmental disorders.

Sakae G. Mizushima received her master's degree from University of Surrey, UK in 2002, and her PhD from United Graduate School of Child Development, Osaka University, 2016. She worked at the Department of Psychosocial Medicine, National Centre for Child Health and Development from 2005 to 2013 in Tokyo, and the Department of Child and Adolescent Psychological Medicine at University of Fukui Hospital, from 2013 to 2016 in Fukui, Japan. Currently, she works at Dokkyo Medical University Saitama Medical Center, Child Development and Psychosomatic Medicine center as a researcher/senior clinical psychologist. Her research interest focuses on developmental psychopathology, using fNIRS, especially, child maltreatment area.

Takeshi Inoue received his medical degree in Japan (2006) and his PhD from Dokkyo Medical University, Japan (2014). His major professional achievements include being a Research Fellow at the Hospital for Sick Children, Toronto, Canada (2017 to 2019) and a Research Associate at the Dokkyo Saitama Medical Center from 2011 to present. He received Board Certified Member of the Japanese Society of Child Neurology (2013).

Keiichi Shimamura received his medical degree in Japan in 2001. He has worked at Dokkyo Medical University Saitama Medical Center, Child Development and Psychosomatic Medicine Center, Japan as a resident and an assistant professor since 2001.

Yuta Ujiie received his PhD from Chiba University Japan, in 2016. He has been a research associate in Research and Development Initiative, Chuo University, since 2016. He has also been a Research Fellow of Japan Society for the Promotion of Science since 2019. His research focuses on the developmental trajectory of multisensory perception and its individual differences.

Akari Arakawa received her medical degree in Japan (2008). Her major professional achievements include being a Research Fellow at the at the Dokkyo Saitama Medical Center from 2014 to present.

Chie Kuroiwa received her master's degree from Bunkyo University in 2014. She has worked at Dokkyo Medical University, Child Development and Psychosomatic Medicine Center, Japan as a clinical psychotherapist since 2014.

Mayuko Ishijima was a research assistant in Jichi Medical University, Japan, and had engaged fNIRS research from 2015 to 2018.

Yuki Kishimoto was a research assistant in Jichi Medical University, Japan, and she currently works at Chuo University, Japan. She has engaged fNIRS research since 2018.

So Kanazawa received $\mathrm{PhD}$ from Kyoto University, Japan in 1997. He was a lecturer and an associate professor at Shukutoku University. Currently, he is a professor in Department of Psychology, Japan Women's University, since 2013. He has authored more than 100 peerreviewed articles. He focuses on early development of perception and cognition in infants and children.

Takanori Yamagata received his medical degree in Japan in 1986 and his $\mathrm{PhD}$ from Jichi Medical University, Japan, in 1993. He was a research associate in the Department of Molecular and Human Genetics at Baylor College of Medicine, from 1997 to 2000. He is currently a professor in the Department of Pediatrics, Jichi Medical University, Japan (since 2011). His research interests include studies on the molecular pathogenesis of autism spectrum disorder, one of the neurodevelopmental disorders. 
Masami K. Yamaguchi received her PhD from Ochanomizu University, Japan in 1995. She was a research fellow in Advanced Telecommunications Research Institute International and an associate professor at Fukushima University and Chuo University, Japan. She is currently a professor in Chuo University, Japan since 2007, and a principal investigator of the MEXT Grant-in-Aid Scientific Research on Innovative Area "Construction of the Face-Body Studies in Transcultural Conditions" since 2017. She has authored more than 100 peer-reviewed articles. Her research interests are perceptual and cognitive development in infants and children.

Ryoichi Sakuta received his medical degree in Japan in 1982 and his PhD from Nihon University, Japan, in 1990. He was a research fellow in the Department of Neuropathology at Toronto Children's Hospital in 2002. He is currently a professor in the Child Development and Psychosomatic Medicine Center, Dokkyo Medical University Saitama Medical Center, in Japan (since 2009). His research interests include studies on neuroscience of child neurodevelopment disorders and psychosomatic diseases such as ASD, ADHD, anorexia nervosa, and gaming disorders.

Ippeita Dan received his PhD from the University of Tokyo, Japan, in 2002. He was a senior research fellow at the National Food Research Institute and an associate professor at Jichi Medical University, Japan. He was appointed as a professor at Chuo University, Japan. He has authored more than 100 peer-reviewed articles attracting about 7000 citations. His research missions lie in clinical applications of fNIRS, methodological development of fNIRS data analyses, and psychometrics applications for business marketing (e.g., food manufacturing). He has served as a director on the board of the Society of fNIRS since 2016. 